\title{
Scope of practice: a statement of the American College of Medical Genetics and Genomics (ACMG)
}

\author{
ACMG Board of Directors ${ }^{1}$
}

Disclaimer: These recommendations are designed primarily as an educational resource for medical geneticists and other healthcare providers to help them provide quality medical genetics services. Adherence to these recommendations does not necessarily ensure a successful medical outcome. These recommendations should not be considered inclusive of all proper procedures and tests or exclusive of other procedures and tests that are reasonably directed to obtaining the same results. In determining the propriety of any specific procedure or test, geneticists and other clinicians should apply their own professional judgment to the specific clinical circumstances presented by the individual patient or specimen. It may be prudent, however, to document in the patient's record the rationale for any significant deviation from these recommendations.

Genet Med advance online publication 23 July 2015

Key Words: clinical laboratory geneticist; clinical laboratory genetics practice; medical geneticist; medical genetics practice; scope of practice
This document defines the Scope of Practice for the primary specialty of Medical Genetics. It serves as a revision of a 2008 American College of Medical Genetics and Genomics (ACMG) Policy Statement (Scope of Practice, retired; available by special request to acmg@acmg.net), which is being updated to clearly delineate (i) the evolving role of the specialty of Medical Genetics and (ii) the distinction between Medical Geneticists and other genetic health-care professionals. Medical Genetics is the application of the principles of inheritance and our knowledge of human genes to diagnose, prevent, and treat diseases and improve health. It is an increasingly broad and unique specialty of medicine that encompasses all organ systems, all periods of life, and many disease entities. Medical genomics is the study of the genome in humans and the relationship of genes and environment in disease. The Medical Geneticist focuses on conditions that are or may be heritable or those that arise from a specific change at the somatic level (for example, cancer cells).

The American College of Medical Genetics and Genomics is the specialty society for American Board of Medical Genetics and Genomics (ABMGG) diplomates and others, providing leadership and resources to facilitate the delivery of high-quality clinical and laboratory medical genetics services. Medical geneticists are certified by the ABMGG, one of the 24 member boards of the American Board of Medical Specialties. The specialty of Medical Genetics includes Clinical Geneticists (MD/DO or equivalent), Medical Geneticists $(\mathrm{PhD})$ and Clinical Laboratory Geneticists (MD/DO or $\mathrm{PhD}$ or equivalent), as well as the clinical subspecialty of Medical Biochemical Genetics (MD/DO or equivalent). The ABMGG also ensures that high standards of continuous education and minimum proficiency are maintained throughout the course of the professional careers of its certified diplomates. For information about specific education and training requirements for each specialty and subspecialty, please refer to the ABMGG website (http://www.abmgg.org).

\section{THE SCOPE OF PRACTICE OF THE SPECIALTY OF MEDICAL GENETICS}

The scope of practice of the specialty of medical genetics includes:

- Genetics consultations, in both inpatient and outpatient settings, with the goal of incorporating available information to make a diagnosis or provide a risk assessment

- Genetic counseling (as described below) for the patient and/or family, including the bidirectional flow of information that includes ethical, cultural, religious, and other implications of such information to allow for decision making by the patient and/or family

- Treatment of genetic diseases, involvement in clinical trials, and natural history studies leading to approval and use of new, orphan drugs and drugs with specific molecular targets

- Early detection and prevention of genetic diseases or their complications through risk-appropriate surveillance and disease prevention recommendations

- Performing genetic and genomic testing, interpreting such results, and providing these results to physicians to facilitate diagnosis, management, and treatment 
- Roles outside of direct patient care, including public health administration, health-professional education, and research. Most Medical Geneticists hold faculty positions and are involved in educational activities in undergraduate and graduate medical education and other graduate education activities

\section{Clinical Geneticists (MD or DO or equivalent)}

The medical practice of Clinical Genetics is based on understanding genetic mechanisms that impact health and disease at both a cellular and an organ-system level. Clinical Geneticists provide consultations, a coordinated clinical service that benefits specialists from every field of medicine, including primary-care providers. A genetics consultation typically consists of a medical evaluation, genetic risk assessment, and genetic counseling and education that together allow for a shared decision-making process between the Clinical Geneticist-led team and the patient and/or family. The medical evaluation consists of multiple components, including a physical examination of the patient or other family members (in a diagnostic setting), risk assessment, proper ordering and utilization of genetic tests, interpretation of genetic test results in the clinical context, and management of inherited conditions. In the evolving practice of genomic medicine, management of inherited risk through prevention strategies and clinical assessment and interpretation of genomic variants is an especially important part of a Clinical Geneticist's practice and requires a high level of content knowledge of genetic and genomic variation.

Often, Clinical Geneticists are also board-certified in other medical specialties and thus have additional responsibilities and expertise related to their training. Similarly, some Clinical Geneticists are also board-certified in a Clinical Laboratory specialty (Clinical Biochemical Genetics, Clinical Cytogenetics, or Clinical Molecular Genetics), and therefore serve as Medical Laboratory Directors and interpret genetic and genomic test results. In the event the provision of genetic health care includes additional members of the genetic health-care team, the Clinical Geneticist is uniquely qualified and trained to assume overall responsibility for the genetic health care of the patient and, sometimes, other family members.

\section{Medical Biochemical Geneticists (MD or DO or equivalent)}

The expansion of newborn screening and the potential to improve outcomes for individuals diagnosed with inborn errors of metabolism created a need for a clinical geneticist with additional subspecialty training in the diagnosis and long-term management of individuals with inherited metabolic diseases. Medical Biochemical Geneticists provide comprehensive diagnosis, life-long management, and genetic counseling services for patients with inborn errors of metabolism, including genetic disorders of intermediary metabolism, lysosomal storage diseases, disorders of energy metabolism, and related disorders. They use their knowledge of the natural history of the disorder, ordering and interpreting specialized laboratory testing in the provision of such services. They interact with other health-care professionals, especially metabolic dietitians, to provide nutritional recommendations for chronic and acute management of individuals with inborn errors of metabolism. They may be involved in the development of new treatments through research and enrolling patients in clinical trials. The Medical Biochemical Geneticist is uniquely qualified and trained to function as the medical home for the metabolic health care of the patient. This subspecialty differs from the primary specialty of Clinical Biochemical Genetics in that Medical Biochemical Genetics training does not provide the educational experience necessary to become a director of a clinical biochemical genetics laboratory.

\section{Clinical Biochemical Geneticists, Clinical Cytogeneticists, and Clinical Molecular Geneticists (MD, DO, PhD, or equivalent)}

Clinical Biochemical Geneticists, Clinical Cytogeneticists and Clinical Molecular Geneticists often serve as directors of CLIA-certified clinical laboratories and are qualified to evaluate laboratory test orders and results within the context of clinical findings and family history, allowing them to provide genetic and genomic test results to physicians to facilitate diagnosis, management, and treatment. This includes interpretation of such results and recommendations for additional testing and genetic counseling. Some individuals are ABMGGcertified in more than one laboratory specialty and have multiple laboratory leadership responsibilities. Historically, some Clinical Biochemical Geneticists were physicians who became board-certified in only this specialty and therefore currently still provide direct patient care and manage patients with metabolic disorders, similar to the roles outlined for the Medical Biochemical Geneticist.

Clinical Laboratory Geneticists are distinguished from other providers of laboratory testing by their specialized clinical fellowship training and certification through ABMGG. Their training is directly applicable to both heritable disease testing and genetic and genomic testing for pharmacogenomics and acquired conditions (such as the somatic genomic changes seen in tumor cells). These uniquely trained laboratory specialists are responsible for oversight and management of their clinical laboratory staff to ensure that accurate genetic/genomic test results are provided to physicians and patients. They must ensure that clinical genetic and genomic laboratory tests are performed in accordance with all applicable rules and regulations, as required. They ensure that standards and guidelines for laboratory procedures, such as the American College of Medical Genetics and Genomics Technical Standards and Guidelines for Clinical Genetics Laboratories, are followed. The combination of providing accurate laboratory results and reporting and interpreting such results by an ABMGG-certified laboratory director, and integration and clinical interpretation of these results in the context of a patient's phenotype by the Clinical Geneticist provides the best opportunity to establish a patient's diagnosis or assess a patient's risk and implement appropriate management. 


\section{PhD Medical Geneticists}

Diplomates with this specialty certification possess broad knowledge of human and medical genetics, including an understanding of genetic disorders and the uses, limitations, interpretation, and significance of specialized laboratory and clinical procedures and medical genetics principles. The PhD Medical Geneticist serves as a member of the Clinical Geneticist-led team, constructing pedigrees, integrating clinical and genetic information (including laboratory test results, such as exome or genome sequence data), family history, and findings from diagnostic evaluations performed by a physician; and providing genetic counseling. In addition, a PhD Medical Geneticist may perform complex risk assessments and paternity and forensic computations.

\section{DELIVERY OF GENETIC AND GENOMIC HEALTH CARE}

The delivery of genetic and genomic health care often requires the combined knowledge of the various specialists discussed above. A genetics evaluation is a medical process that results in clinical decision making, typically involving a differential diagnosis, genetic risk assessment, testing strategy (including ordering and interpreting the results of genetic and genomic tests in the context of a patient's phenotype), and recommendations specific to the genetic risk for that patient or diagnosis. The Clinical Geneticist is responsible for all components of the genetics evaluation, although a trained and supervised nonphysician genetic health-care professional (e.g., genetic counselor, nurse, $\mathrm{PhD}$ Medical Geneticist) may perform some components of the evaluation (e.g., family history documentation, pedigree analysis, review of records, genetic counseling, explanation of genetic and genomic test results). Genetic counseling is a bidirectional communication process that facilitates patient decision making surrounding the genetics evaluation; as such, it requires input from the clinician. Taken together, the genetics evaluation and genetic counseling allow for a shared decision-making process between the clinical geneticist-led team and the patient and/or family. The Clinical Laboratory Geneticist provides diagnostic test results and interpretation necessary to establish a diagnosis. The Medical or Clinical Biochemical Geneticist provides additional expertise and management for a patient with an inborn error of metabolism. After a consultation, the Clinical Geneticist often remains involved as a key member of a patient or family's health-care team and may serve as the patient's medical home since they are best acquainted with the related medical issues, longitudinal care, and anticipatory guidance.

As evidenced above, the Scope of Practice of the Medical Geneticist is broad and includes direct patient care, genetic counseling, education, and laboratory medicine. The primary specialty of Medical Genetics continues to evolve as new disorders are identified, new technologies become available, and new treatments become the standard of care. As such, the American College of Medical Genetics and Genomics Scope of Practice will continually be adapted to meet the needs of the patients served by Medical Geneticists.

\section{DISCLOSURE}

The authors declare no conflict of interest. 